\title{
Ueber die Entwicklungsgeschichte und die Anatomie von Gordius tolosanus Duj. $=$ G. subbifurcus v. Siebold ${ }^{1}$ ). Von
}

Dr. v. LIustow in Göttingen.

Hierzu Tafel XIV, XV, XVI.

Unsere Kenntniss des merkwüirdigen Genus Gordius verdanken wir vorwiegend v.Siebold, Me is s ner, Schneider, Grena c ber, Villot, Vejdorsky und Camerano, durch deren Studien wir uber die Anatomie gut orientirt sind, wenngleich auch hier noch manche Widersprtiche auszugleichen sind; anders steht es mit der Entwicklungsgeschichte; mit derselben hat sich vorwiegend Villot beschäftigt und ist dabei zu dem Resultate gekommen, dass die Gordien tiberhaupt keine speciellen Zwischenwirthe haben.

Bevor ein so allgemeiner Satz aufgestellt wird, scheint es mir nöthig, die Entwicklungsgeschichte der einzelnen Arten zu studiren, und bin ich in der Lage, $\mathrm{z}$ der von Gordius tolosanus Duj. einen Beitrag zu liefern; ich bin weit entfernt, auf Grund der hier mitgetheilten Beobachtungen die Entwicklungsgeschichte des ganzen Genus Gordius erklären zu wollen; namentlich weiss ich nicht, wie Gordius-Larven, die in Schmetterlingsraupen gefunden sind, in diese hinein und später aus ihnen in's Wasser gelangen; vor allgemeinen Schliussen werden wir uns vorläufig um so mehr zu hüten haben, als wir zur Zeit nur die Zwischenwirthe für beide Larvenformen von Gordius aquaticus und tolosanus kennen.

1) Vill ot, Revision des Gordiens, Ann. sc. natur. 7. sér., t. I, 1886, art. Nr. 5, pag. 296-302. 
Die erste oder embryonale, d. h. dem Embryo ähnliche Larvenform von Gordius tolosanus wurde von Meissne ${ }^{1}$ ) in Larven von Ephemera, von Villot ${ }^{2}$ ) in Larven von Tanypus, Corethra und Chironomus beobachtet; die zweite, grosse Larvenform wurde in Käfern, vorwiegend in Lanfkifern gefunden und zwar in Carabus hortensis Fabr., Procerus (Carabus) coriacens Lin., Calathus fuscipes Goeze = cisteloïdes Panzer, Poecilus lepidus Fabr., Molops elatus Fabr., Pterostichus metallicus Fabr., Pterostichus (Omaseus) vulgaris Lin., Pterostichus (Omaseus) melas Creutzer, Pterostichus (Omaseus) nigritus Fabr., Harpalus atratus Latr. = hottentotta Duftschmidt, Amara similata Gyll., Calathus ambiguus Payk., Amara fusea Sturm, Zabrus (Pelor), blaptoïdes Creutz. und Silpha carinata Illig.

Herr Geheimrath Professor Dr. Ehlers batte die Gute, mir Gordien mitzutheilen, die im Sommer 1888 in der Nähe von Göttingen in einem kleinen Bache in Menge gefunden waren; sie liessen sich als zu Gordius tolosanus gehörig bestimmen und besuchte ich im Frubling 1889 den Fundort, um mich für spätere Excursionen zu orientiren, als meine Aufmerksamkeit auf Käfer gelenkt wurde, die theils todt, theils sterbend, theils noch lebend im Bache lagen, einige frei schwimmend, andere an Pflanzen angeklammert oder in Algenmassen verwickelt; in der Zeit einer Viertelstunde sammelte ich 18 Exemplare, an einem der nächsten Tage in einem benachbarten Bache 14, die sich als zu Pterostichus niger Schaller gehörig erwiesen, nur 1 Exemplar wurde als Harpalus hirtipes Panzer bestimmt. Die Käfer wurden geöffnet und in einem Pterostichus niger fand sich ein $115 \mathrm{~mm}$ langes und $0,48 \mathrm{~mm}$ breites weibliches, in einem anderen ein $122 \mathrm{~mm}$ langes männliches Exemplar von Gordius tolosanus; sie Iagen lockenförmig aufigerollt in der Leibeshöhle des Käfers, waren hellbraun von Farbe und bewegten sich nach dem Freiwerden lebhaft.

Räthselhaft scheint es, wie die Käfer in den Bach gerathen waren; eine nahe liegende Veranlassung dazu fehlte vollstïndig; es war kein Gewitterregen vorbergegangen, eine Ueberschwemmung batte nicht stattgefunden; auch waren diese Käfer die einzigen in's Wasser gerathenen Landthiere.

1) Zeitschr. für wissensch. Zoolog. VII, Leipzig 1856, pag. 131, tab. VII.

2) Arch. zool. expériment. III, Paris 1874. 
In Bezug auf diese Gordiuslarven theilte Herr Geheimrath Ehlers mir mit, dass dergleichen Funde bei Giottingen schon frtiber gemacht seien, und befinden sich in der Sammlung des hiesigen zoologischen Instituts Larven von Gordius tolosanus aus Procrustes coriaceus Lin., Pterostichus niger Schall. und Calathus cisteloïdes Panz., sowie unbestimmte Gordius-Larven aus Calathus cisteloïdes Panz., Calathus (Harpalus) ruficornis Goeze und Silpha atrata $L$.

Die Entwicklung dieser Art würde somit als festgestellt angenommen werden können, wenn nicht Villot, der sich sehr eingebend mit dem Genus Gordius beschäftigt hat, zu ganz abweichenden Resultaten gekommen wäre.

Vill ot stellt in seiner grossen Arbeit, der Monographie des Dragonneaux ${ }^{1}$ ) die Entwicklungsgeschichte so dar, dass die kleinen, embryonalen Gordius-Larven, speciell die von Gordius aquaticus, tolosanus, und grationopolensis, im Sommer in Larven von Tranypus, Corethra und Chironomus gelangen, um sich hier zu encystiren, dass diese Dipteren-Larven ron kleinen Fischen, Phoxinus laevis und Cobitis barbatula gefressen werden, wodurch die Gordien aus ihren Cysten befreit werden und sich in die Darmwand der Fische einbohren, um sich hier auf's neue zu encystiren und so den Winter über zu verharren. Im nächsten Frubling bersten die Kapseln, die Gordien gerathen frei in das Darmlumen und mit den Excrementen in's Wasser, wo sie zu den bekannten, grossen Formen auswachsen und geschlechtsreif werden. In einer späteren Arbeit ${ }^{2}$ ) modificirt Villot seine Ansicht dahin, dass er meint, die beiden Phasen des Parasitismus der Gordien verliefen in einem und demselben Wirth.

$\mathrm{V}$ ill ot findet die embryonale Larvenform eneystirt in der Darmwand der genannten Fische und in Petromyzon Planeri, in den angefubrten Dipteren-Larven, ferner in Hydrophilus piceus, in Planorbis, er verzeichnet das Vorkummen nach Leydig in Rana temporaria, nach Claparède in Enchytraeus vermicularis, nach Meissner in Ephemera, nach mir in Limnaeus vulgaris; die zweite, grosse Larvenform aber ist gefunden in Coleopteren,

1) 1. c.

2) Développement des Gordiens, Ann. sc. natur., 6 sér., t. XI, art. 3, 1881, pag. 18-20. 
Orthopteren, Neuropteren, Hymenopteren, Lepidopteren, Dipteren, Hemipteren, in Arachniden, in Crustaceen, in Fischen (Cobitis, Petromyzon, Thymallus, Aspius, Coregonus, Salmo), in Amphibien (Rana), in Vögeln (Otis) und im Menschen. So kommt Villot zu dem Schluss: "Les Gordiens u'ont pas d'hôtes spéciaux"; das in sehr zahlreichen Fillen, besonders von v. Siebold angefithrte Vorkommen der zweiten, grossen Larvenform in Insekten nennt or eine anomalie d'uabitat und bezweifelt die richtige Bestimmung der Helminthen, ein Einwand, den ich, da dieselbe von v. Siebold herruhrt, nicht zugeben kann; bei den ron mir in Pterostichus gefundenen Exemplaren kann ich dafür einstehen, dass es sich um Gordius tolosanus bandelt. In seiner neuen Arbeit, Anatomie des Gordiens, giebt Villot an, selber zahlreiche Larven von Gordius violaceus in 5 Exemplaren von Procrustes coriaceus gefunden zu haben, ohne sich weiter auf die Tragweite und Deutung dicses Fundes einzulassen.

Bei der Besprechung der grossen Larven von Gordins tolosanus aus Käfern erwähnt $\mathrm{V}$ ill o $t^{1}$ ) als einen besonders interessanten Fall des Auffinden eines Exemplars im Darm eines Menschen durch Fiori in Piemont, mitgetheilt von Rosa, and meint, während er das Vorkommen in Käfern für eine anomalie d'habitat hïlt, ein solches Vorkommen beruhe nicht auf einem Pseudoparasitismus; die Piemontesen ässen keine Käfer, vielmehr müsse ein Gordius-Embryo mit dem Trinkwasser in den Darm gekommen sein, wo er sich als wahrer Parasit entwickelt habe, ebensogut, wie er es in einem Käfer oder einer Spinne gethan haben wuirde.

Gordius aquaticus ist im Mensehen gefunden von Aldrovandus, Degland, v. Siebold und von v. Patruban (nach Villot war der von Degland beobachtete Gordius ein G. tolosanus); der von Fiori mitgetheilte Fall bezieht sich auf Gordius tolosanus; Kirkland fand nach Diesing Gordius varius, Gay Gordius chilensis und Cerruti und Camerano Gordius Vilotti, Pavesi Gordius Villoti und tolosanus im Menschen; Bacounin berichtet von absichtlich verschluckten Gordien, uber welche Beobachtung Cerruti und Camerano sagen: "che questi vermi non resistono ad una temperatura di 38 gradi e di piu essi non pag. 301 .

1) Revision des Gordiens, Ann. sc. natur., 7. sêr., t. I, art. 5, 1886, 
diedero mai luogo al piu piccolo malessere essendo stati perfettemente digeriti“. Da Gordien schon aus Brunnen geschöpft wurden, so bat v. Siebold ${ }^{1}$ ) gewiss recht, wenn er als selbstverständlich annimmt, dass das Vorkommen von Gordien im Menschen die Folge von zufälligem Verschlucken derselben mit Trinkwasser ist. Mir wurde einst aus einem Soldaten-Wachtlokal eine Wasserflasche gebracht, mit der Frage, was fïr ein Thier darin sei; es bewegte sich ein Gordius aquaticus im Wasser, und wenn einer der Soldaten in der Nacht aus dieser Flasche getrunken hätte, wäre es nur zu leicht möglich gewesen, dass er dẹn Gordius mit verschluckt hätte. Die in Fischen und Vögeln beobachteten grossen Larven werden aus mit solchen Larven inficirten Insekten stammen, die ersteren zur Nahrung dienten, denn alle genannten Fische und Vögel sind Insektenfresser; kein Wunder, dass sie dann auch einmal gelegentlich mit einem Insekt eine GordiusLarve verschlingen, die dann vermuthlich entweder verdaut oder mit den Excrementen entfernt wird.

Dass sich Gordius-Embryonen derselben Art gleicherweise in Insekten, in kalt- und in warmblitigen Wirbelthieren entwickeln sollen, halte ich nach unseren anderweitigen helminthologischen Erfahrungen für undenkbar, und aus den gesammten Funden von Gordius-Larven ohne Rïcksicht auf die Gordius-Arten und ohne die Fälle auszuscheiden, welche als Pseudoparasitismus gelten mï̌ssen, den Schluss zu ziehen: „Les Gordiens n'ont pas d'bôtes spéciaux" halte ich für ebenso unbegründet, als wenn man dasselbe von den Cestoden behaupten wollte, deren Larven auch in Sïugethieren, Vögeln, Fischen, Insekten, Myriapoden, Crustaceen, Mollusken und Helminthen gefunden werden.

Für Gordius tolosanus muss ich den Entwicklungsmodus annehmen, dass die kleinen, embryonalen Larven eingekapselt in Wasserlarven ron Ephemera, Corethra, Chironomus und Tanypus, die grossen aber frei in der Leibeshöhle von Laufkïfern leben, welehe im Fruhling ins Wasser fallen, wodurch die Gordien wieder in ibr eigentliches Element gelangen.

Das Hineingerathen in die auf dem Lande lebenden Käfer wie das Herauskommen aus deuselben in's Wasser ist in gleicher Weise merkwürdig. Ersteres wird im Spätsommer geschchen,

1) Zeitschr. f. wissensch. Zonlog. VII, Leipzig 185it, pag. 142. 
wenn Theile von Bäcben und Teichen ausgetrocknet sind; so dass die Käfer so die Wasserlarven der Dipteren erlangen und fressen können, welche die embryonale Larvenform beherbergen; während des Winters wachsen die jungen Gordien dann in den Käfern Leran. Das massenhafte Ertrinken der Laufkafer im Frühling ist das Mittel, durch welches die grosse, zweite Larvenform wieder in's Vasser gelangrt; dasselbe kamn aber wohl nur darin seinen Grund haben, dass die Käfer in dieser Jabreszeit auf dem Lande noch keine Beute finden, während das Thierleben im Wasser schon erwacht ist und Sclmecken eine Lieblingsnahrung der Laufk̋̈er bilden, so dass sie, wïlurend sie am Rande eines Baches oder 'Teiches ihrer Nahrung nachgehen, in Menge ihren Tod dureb Ertrinken finden.

Die Zeit, zu welcher die Gordius-Larven mit den Laufk:ifern in's Wasser gerathen, ist der Monat April; Ende Juni findet man im Wasser schon geschlechtsreife Exemplare. In den betreffenden Bächen, in welchen ich die Funde machte, kommen Gasterosteus aculeatus und pungitius, Cottus gobio, Gobio fluviatilis, Cobitis barbatula und Phoxinus laevis häufig vor, niemals aber fand ich Gordien irgend einer Entwicklungsstufe in ihnen.

\section{Anatomie der Larven ans Kälern.}

Die ans Käfern erhaltenen Larven benutzte ich, die Anatomie derselben zu studiren, und hattc ich über dreierlei Präparate zu verfligen, nämlich über vor einigen Jahren im hiesigen zoologisehen Institut gemachte Serienschnitte einer ans Procrustes coriaceus stammenden Larve, welche Herr Geheimrath Ehlers die grosse Liebenswirdigkeit hatte, mir zur Benutzung zu uberlassen; über Serienschnitte der von mir gefundenen Exemplare aus Pterostichus niger, welche Herr Dr. Hamann treundlicher Weise anfertigte, und uber selbstgemachte von denselben Exemplaren.

Die $\mathrm{C} u$ t is (Derma) ist 0,013 mm dick und aussen von der Epidermis bedeckt; erstere ist hellbraun und faserig, letztere viel dunner, dunkelbraun und an der Aussenseite durch dichtgedrängte, pflastersteinartige Erhebungen ausgezeichnet, wie sie für die Art charakteristisch sind. Vier Schichten, wie Camerano ${ }^{1}$ ) sie als

1) Ricerche intorno alla anatomia ed istologia dei Gordii. Torino 1888. 
an einzelnen Stellen vorkommend schildert, nämlich 1 . strato cuticolare esterno, 2. straterello interotto di stostanza granulosa, 3. strato fibrillare und 4. straterello interotto di granulazionie finden sich bei den ron mir untersuchten Larven nicht, sondern nur die Schichten 1 und 3 . Vejdovsky ${ }^{1}$ ) nennt die beiden Schichten die homogene und die faserige Cuticula und bemerkt, dass die erstere an den beiden äussersten Körperenden glatt, durchsichtig und farblos wird. Meissner bezeichnet die beiden Schichten mit Epidermis und Corium, Villot mit Epiderme und Derme, später mit couche superficielle und profonde der cuticule. Die beiden Schichten sind bei den Larven bereits ebenso deutlich gesondert wie bei den freilebenden Exemplareu, entbehren aber noch der Kreuze mit den Poren und der Sinnesborsten.

Die Hypodermis ist eine $0,0066 \mathrm{~mm}$ dicke, aus gekernten, polygonalen Zellen bestehende Schicht unter der Cutis; am Kopfund Schwanzende schimmern die Kerne durch die transparente Cutis hindurch; in der Scheitelgegend ist sie stark verdickt und beim Männchen erhebt sie sich leistenförmig in der Bauchlinie, die Muskulatur theilend; da, wo am männlichen Schwanzende die Theilung beginnt, ist sie ebenfalls verdickt (Fig. 11), bildet in der Gegend der vorderen Schwanzganglien dicht hinter der Cloakenöffnung die Aussenwand des Körpers, da hier die Cutis fehlt, und macht vor der Gabelung eine von der Ritcken- nach der Bauchlinie ziehende Brïcke (Fig. 13); in den beiden Schwanzgabeln ist sie in der Bauchseite wesentlich verbreitert (Fig. 14). Beim Weibchen ist sie in der Gegend des Uterus an der Baucbseite etwa 5 mal dicker als an der Rückenseite (Fig. 19); die Kerne sind entweder ei- oder kugelförmig. Meissner bezeichnet die Hypodermis als Perimysium, während Grenacher ${ }^{2}$ ) sie subcutane Schicht nennt und sie als Matrix der Cutis hinstellt. Villot hat die seltsame Ansicht, die Hypodermis, welche weder aus epithelialen Zellen noch aus einem gekernten Protoplasma, sondern aus fibrillären Elementen bestehe, sei ein Theil des Nerrensystems, eine Ansicht, welche er auch in seiner neueren Arbeit "Sur l'ana-

1) Zur Morphologie der Gordiiden. Zeitschr. für wissensch. Zoolog. XLIII. Leipzig 1886.

2) Zeitschr. für wissensch. Zoolog. XVIII, Leipzig 1868. 
tomie des Gordiens “ I) festhält. Der Bauch-Nervenstrang soll mit der Hypodermis unmittelbar zusammenbängen. Wäre diese Auffassung richtig, so wiirden wir ein Nervensystem bei Gordius vor uns haben, das unmittelbar der Cutis anliegend cylinderförmig den gauzen Körper überzieht und an einer Stelle, dicht hinter der Cloake des Männchens, frei zu Tage liegt. Ganz neuerdings erklärt Villot ${ }^{2}$ ) die Hypodermis für ein Gefässsystem, das eine Absorbtions- oder Excretionsfunction besitzt, während ausserdem ein Netzwerk von Ganglienzellen zwischen der subcutanen Schicht und dem Perinysium liegen soll. Vejdovsky ${ }^{3}$ ) nennt die Hypodermis eine cuticularbildende Matrix, die in ihren verschiedonen Gestaltungsverhïltnissen immer von einer Epithelschicht ableitbar ist. Letzterer Umstand hat Camerano veranlasst, das Gebilde nicht Hypodermis, sondern Epidermis zu nennen, eine

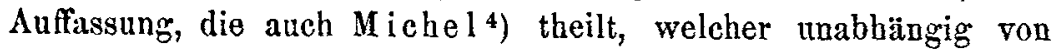
erstcrem die zellige Natur erkannte. Sowohl Camerano wie Michel scheint es entgangen zu sein, dass ich ${ }^{5}$ ) bereits im Jahre 1877 die zellige Natur der Hypodermis beschrieben und abgebildet habe; meine Angaben wurden aber von späteren Forschern ange\%weifelt; die Kenntniss der zelligen Structur ist übrigens noch viel ailter, denn sie war sehon Schneider bekannt, der in seinen ${ }_{n}$ Untersuchungen uber Gordius" Fig. $17 \mathrm{c}$, die zellige Hypodermis, die er, wie bemerkt, Perimysium nennt, abbildet.

Was die Frage betrifft, ob das betreffende Stratum Hypodermis oder Epidermis zu benennen ist, so kann von beiden Bezeichnungen wohl nur in Bezug auf ein Derma die Rede sein; nennt man es Epidermis, so ist die nach aussen von ihm liegende Sehicht die Cuticula, und sieht man sich alsdann vergebens nach einem Derma um, denn die unmittelhar darmnter liegende Schicht von Längsmuskeln kann doch nicht als Derma bezeichnet werden. Das fragliche Stratum scheint mir aber der ebenfalls aus polygonalen Zellen bestehenden Hypodermis oder Matrix oder Chitinogen$304-306$.

1) Ann. sc. natur. zoolog. 1887, art. 4, pag. 193.

2) Compt. rend. Acad. sc. Paris, f. CVIII, Nr. 6, Paris 1889, pag.

3) 1. c. pag. 380 .

4) Compt. rend. Acad. sc. Paris, vol. CVII, pag. 1175-1177.

5) Archiv für Naturgesch. 1877, pag. 3-4, tab. I, Fig. 5. 
Membran der Arthropoden homolog zu sein, daher ich es als $\mathrm{Hy}$ podermis bezeichnen möchte; die nach aussen von ihm liegende Schicht wäre alsdann Cutis oder Derma und die dunkelbranne, dünnere Aussenschicht Epidermis zu benennen.

Die Muskeln liegen der Innenseite der Hypodermis an; sie bestehen lediglich aus Längsmuskeln und erreichen in der männlichen Larve eine Dicke von $0,051 \mathrm{~mm}=1 / 6$ des Körperdurchmessers, in der weiblichen eine von $0,029 \mathrm{~mm}=1 / 12$ desselben; etwa $0,49 \mathrm{~mm}$ vom Kopfende entfernt beginnen sie mit einer anfangs sebr dünnen, dann bald mäehtiger werdenden Schicht. Die langen Muskelzellen haben einen gestreckten, stabförmigen Kern, wie man an Längsschnitten sieht (Fig. $21 \mathrm{~d}$ ). $\Lambda \mathrm{m}$ männlichen Schwanzende theilt sich die Muskelmasse, die bei beiden Geschlechtern in der Baucblinie durch den Verbindungsstrang zwischen Hypodermis und Nervenstrang unterbrochen ist, in zwei seitliche Hälften, und wenn die Gabelung vollendet ist, findet man die Muskulatur beschränkt auf das innere, der Rückenseite anliegende Viertel und die äussern zwei Drittel (Fig. 14). Das männliche Schwanzende zeigt ausserdem an der Stelle, wo der Darm sich nach der Rückenseite wendet, zwei dorsoventrale Muskelzüge (Fig. 10), die sich dicht hinter der Cloake zu einer starken Masse vereinigen (Fig. 11 und 12), von welchen Muskeln Vill ot ${ }^{1}$ ) irrthïmlich behauptet, dass sie aus Parenchym-Elementen bestehen. Am weiblichen Schwanzende ist in der Gegend des Uterus die Muskulatur an der Bauchseite erheblich duinner als an der Rückenseite (Fig. 17), schwindet an ersterer bald ganz (Fig. 18 und 19) und hat bei der Cloakennüundung völlig anfgehiört (Fig. 20). Jede Muskelfibrille enthält an der Innenseite einen Kern, der in die Marksubstanz übergebt und nach aussen stark verduinnt ist (Fig. 24); letztere liegt an der Aussenseite, welche mit der Hypodermis in Berihrung ist und zeigt auf Quersebnitten eine parallele Begrenzung nach den Seiten; sie ist durch Hämatoxylin gut sichtbar zu machen. Grena (: her ${ }^{2}$ ) erkannte, dass die Muskelfibrillen nicht ununterbrochen vom Kopf- bis zum Schwanzende verlaufen, sondern dass sie $0,5-0,66 \mathrm{~mm}$ lang sind und die

1) Anatomie des Gordiens, pag. $20 \%$.

2) Zeitschr. für wissensch. 'Zoolog. XIX, Leipzig 1849, pag. 289-290, tah. XXIV, Fig. 4. 
Ueb. A. Entwicklangsgesehichte u. d. Anatonie v. Gordius tolusauus Duj. 257

Furm eines Paralleltrapezes haben; el hält die Gordienmuskeln nicht den Nematodenmuskeln für gleichwerthig, da am visceralen Rande eine Höhlung ohne Kern erkennbar sei und da sie beiderseits in eine Spitze auslaufen; das erstere habe ich nicht bestïtigen können. Schneider rechnet die Gordien-Muskeln zur Gruppe der Holomyarier; wenn nun anch die Schneider'sche Diagnose nicht in allen Punkten bestätigt ist, so bilden diese Muskeln doch eiuen scharfen Gegensatz zu den Poly- und Meromyariern, so dass die Schneider'sche Eintheilung trotzdem sehr werthroll erscheint. Was Vejdovsky eine Dorsalfurche in der Muskulatur am miinnlichen Schwanzende nennt, balte ich fiir nichts wciter als die Zeicheu der beginnenden Gabelung; wenn derselbe den Muskelkern der Muskeln von Gordius tolosanus seitlich, ausserhalb der Muskelzelle liegen liisst, so gestehe ich, dass ich derartiges weder bei meinen Larven noch bei geschlechtsreifen Exemplaren gesehen habe.

Der Zellkörper dient theils als Stütze der inneren Organe, theils als Füllsubstanz, wie z. B. in der männlichen Schwanzgabel, theils aber als Bildungskörper für die Hoden und die Ovarien, die beide in der Larve noch nicht vorbanden sind; nach der Körperperipherie zu pflegen die grösseren Zellen zu liegen; niemals habe ich ein Epithel der Leibesböhle gesehen, wie Vejdorsky es beschreibt und abbildet; auffallend ist die segmentirte Anordnung der Zellen, wie sie auf Längsschnitten besonders in der Peripherie des Körpers deutlich ist (Fig. 21). Grenacher nennt den Zellkörper perienterisches Zellgewebe, Schneider MuskelMarksubstanz.

Sowohl die männliche wie die weibliche Larve zeigt auf Querschnitten zwei seitliche, symmetrische, der Rückenfläche genïherte und einen unsymmetrischen, der Banchfläche näher liegenden Hohlraum; in letzterem liegt der Darm und an der Bauchseite grenzt der Nervenstrang an ihn; ich halte ihn für eine Leibeshïble. Sie bietet bei den Larven einen Formunterschied nach den Geschlechtern; beim Mänuchen ist sie nach der Rickenseite zn rundlich begrenzt, auf Querschnitten erscheint sie hier nieren- oder hufeisenförmig. (Fig. $8 \mathrm{~b}$ ), wiihrend sie beim Weibchen nach dem Ritcken zu spitzwinklig begrenzt ist, so dass der

1) 1. c. tab. XV, Fig. $36 \mathrm{pt}$. 
Querschnitt die Form eines Kartenherzens hat (Fig. 16 b). Vejdovsky nennt die Leibeshöhle Excretionscanal; ich habe keinen Grund zu einer solchen Deutung gefunden und kenne keinen Excretioncanal, in dessen Lumen ein Darm verläuft; $V$ illot nennt die Leibeshöhle eavité de regression de l'intestin, in jüngster Zeit aber cavité périintestinale, die durch einen Zerfall der den Darm umgebenden Parenchymzellen entstehen soll ${ }^{1}$ ).

Der Verdau ungtract beginnt mit einer scheitelständigen Oeffnung, welche die Cutis durchsetzt; das daranter liegende Parenchym verlegt aber die nun folgende Röhre (Fig. 7 b), so dass ein Lumen bei den grossen Larven aus Käfern nicht mehr existirt; auffallender Weise ist der dicke Anfangstheil des Oesophagus aus zwei seitlichen, symmetrischen Hälften zusammengesetzt (Fig. 2 und $3 \mathrm{~b}$ ); zunächst verläuft er in der mittleren Körperachse, wird dann daselbst vom Kopfganglion umgeben (Fig. 3 u. 4), verläuft etwas weiter hinten getrennt von letaterem an dessen Rückenseite (Fig. 5) und erscheint auf Querschnitten mehrfächerig (Fig. 6); der der Mundöffnung zunächst liegende Theil ist kelchförmig erweitert (Fig. 7).

Der Darm hat ein deutliches Lumen und wird aus Zellen mit eiförmigen, granulirten Zellen gebildet (Fig. 23) ; beim Männchen münden die Ausführungsgänge der Geschlechtsröhren in das Ende des Darms, eine Cloake darstellend, nachdem der Darm am Schwanzende von de: Bauchlinie nach der Rückenseite getreten ist und darauf, nachdem er wieder zur Bauchlinie zurückgekehrt ist, sich stark von der Rücken- nach der Baucliseite verbreitert hat. Bei beiden Geschlechtern ist das letzte Ende des Darms von stark erweitertem Lumen und sehr verdickten Wänden. Beim Weibchen verläuft der Darm in der Gegend des Uterus nahe der Riückenlinie (Fig. 18 und 19 a), in dessen hinterstes Ende er einmündet, wie Grenacher bereits erkannte. Meissner nennt den Darm Excretionsorgan.

Das Central-Nervens ys tem beginnt unmittelbar hinter der Mundöffnung mit zwei schwachen, neben einander liegenden Anschwellungen (Fig. 2), welche an der Rückenseite des obliterirten Mundbechers liegen, um sich dicht dahinter zu einer grossen $685-687$.

1) Compt. rend. Acad. sc. Paris t. CVIII, Nr. 13, Paris 1889, pag. 
Nervenmasse zu vereinigen, die den Oesophagus von allen Seiten umgiebt (Fig. 3 u. 4); dann tritt sie nach der Bauchfläche hin, nimmt bedentend an Umfang ab und verläuft isolirt vom Oesophagus (Fig. 5 u. 6); anfangs höher als breit (Fig. 5) wird das Organ nun bald breiter als boch (Fig. 6) und besteht aus drei deutlich gesonderten, neben einander liegenden Strängen, die an der Basis von einer gekernten Masse gestiitzt werden; der Bauchstrang trennt die Muskulatur in der Bauchlinie und ist durch einen faserigen Strang mit der Hypodermis verbunden. Vill ot's Auffassung: „Le ganglion céphalique n'est autre chose qu'en renfement de la couche hypodermique ${ }^{\text {" }}$ passt für die von mir untersuchten Larven durchaus nicht (Fig. 3).

Beim Männchen erhebt der Bauchstrang sich dicht vor der Schwanzgabelung von der Muskulatur und bekommt nun eine besondere, seitliche, aus Maskelmasse bestehende Stitze (Fig. 9 u. 10); beim Beginn der Gabelung tritt einer der Seitenstränge an die Innen- und Bauchseite je einer Gabel (Fig. 11), der Mittelstrang schwindet, dicht hinter der Cloakenöffnung bemerkt man in jeder Gabel eine starke Ganglien-Anschwellung (Fig. 12) und nach vollzogener, vollständiger Trennung der Endäste in jeder derselben eine zweite (Fig. 14). In beiden Geschlechtern ist der ungetheilte Bauchstrang von einem gekernten Bindegewebe rings umgeben. Bei der weiblichen Larve hört der Bauchstrang mit dem Uterus auf, eine Theilung findet nicht statt, an der Cloakenöffnung ist er nicht mehr vorhanden (Fig. 20). G r e $\mathrm{n}$ a c h e $\mathrm{r}^{1}$ ) nennt den Bauchnervenstrang Ventrallinie; er lässt denselben auch am Schwanzende des erwachsenen Weibchens sich gabeln ${ }^{2}$ ), was

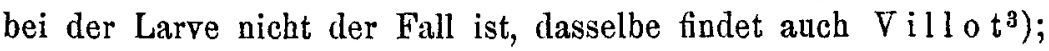
Schneider ${ }^{4}$ ) bezeichnet ihn als Oesophagus.

Ein intermuskuläres oder interparenchymatöses Wassergefässsystem, welches Villot beschreibt, existirt bei den von mir untersuchten Exemplaren nicht, auch $\mathrm{Camerano}$ leugnet die Existenz eines solchen.

Die männlichen Geschlechtsorgane waren in den

1) 1. c. pag. 285 .

2) 1. c. pag. 328 .

3) Anatomie des Gordiens pag. 195.

4) I. c. pag. 185 . 
Larven noch nicht entwickelt; von den Hodeu bemerkt man noch nichts und die beiden erwähnten, nach der Rückenfläche zu gelegenen, symmetrischen Lücken im Zellkörper entsprechen ihrem späteren Lumen; sie sind im Gegensatz zu der Leibeshöhle mit einem Epithel ausgekleidet, das nicht überall der Wandung flach anliegt, sondern zum Theil frei in die Höhlung hineinragt. Nach dem hinteren Körperende verengern sich die Röhren und gehen in die Samenleiter uber, welche links und rechts in den hintersten Theil des Darms treten (Fig. $11 \mathrm{~d}$ ) und so mit ihm die Cloake bilden; eine Muskulatur findet sich an der männlichen Cloakenmitndung nicht. Ausser den kleinen Kegeln, welche die Cloakenoffnung dicht und den zum Theil arn Ende gespaltenen kleinen Borsten, welche sie in weiterem Bogen umgeben, findet sich noch eine dritte Gruppe feiner Spitzen, welche die Innenseite der Endlappen bekleiden.

Die weiblichen Geschlechtsorgane bilden bei geschlechtsreifen Thieren, abgesehen vom vordersten und hintersten Körpertheil fünf parallele Röhren; an der Rückenseite der Rückencanal (Fig. $31 \mathrm{~d}$ ), seitlich davon die Eiersäcke (Fig. $31 \mathrm{c}$ ), nach aussen von ihnen die Ovarien (Fig. 31 a u. b); letztere vier Organe, die beiden Eiersäcke und die beiden Ovarien, grenzen nach der Bauchseite za an die Leibeshöhle (Fig. $31 \mathrm{e}$ ); am Schwanzende gehen die Eiersäcke in die kurzen Eileiter iiber (Fig. 17 a), welche in den Uterus münden, und an dessen Bauchseite liegt das gleichfalls in den Uterus mündende Receptaculum seminis.

In der Larve aus Käfern sind von diesen Organen nur die Eiersäcke und ihre hinteren, kurzen Ausläufer, die Eileiter und der Uterus vorhanden (Fig. 16, 17, 18, 19); Ovarien, Receptaculum seminis und Riickencanal fehlen gänzlich. Die Eiersäcke entsprechen morphologisch den Hodenanlagen der Larve; sie sind, wie diese, Hohlräume und mit einem ähnlichen Epithel ansgekleidlet; die Eileiter sind dickwandig. Der Uterus besteht aus zwei sehr verschiedenen Abschnitten; der vordere ist zweitheilig (Fig. 18 b) und von seiner Innenwand erheben sich pilzförmige Bildungen, während die hintere Hälfte (Fig. 19 b) von einem Netzwerk einzelliger Drisen ansgekleidet ist. Der Durm tritt von der Riickenseite in den allerhintersten Theil des Uterus; eine seitliche Lagerung dem Uterus gegeniuber wie beim erwachsenen Thiere findet 
Veb. A. Eutwicklungsgreschichte u. C. Analomie v. Gordius tolosanus Duj. 261

nicht statt; die so gebildete Cloake ist von einer hypodermisähulichen Membran begrenzt (Fig. 20).

Nacb Grenacher waren bei einer Larve von Gordius ornatus ans Mantis die Eianlagen schon stark entwickelt und im Ovarium polygronale Zellen, die unreifen Eier, vorhanden. Vejdovsky ${ }^{1}$ ) yab in seiner ersten Arbeit an, die wahren Eierstöcke niemals gefunden zu haben; er hatte sie in der That aber wohl gesehen und nar nicht richtig gedentet, denn was er Tab. XVI Fig. $61 \mathrm{eg}$ mit Eileiter bezeichnet, sind Ovarien und die mit $d^{\prime}$ bezeichneten Organe die leeren Eiersïcke, von ilum als Leibeshöhle bezeichnet; in sciner zweiten Arbeit") werden sie als Ovarien angeführt, ihre Hohlrilume aber als Cölom oder Leibeshöhle. Me is s ner nenut dio Ovarien Eierstockschliuche, Vill o t die lateralen Aeste der Ovarion.

Die Eicrsïcke werden von Grenacher als Oviducte bezeichnet, von Meissner als Ovarien, während Vejdovsky sie Eiersäcke und Eierbehälter und Villot die dorsalen Aeste der Ovarien nennt. Letzterer spricht sowohl den Eiersäcken als auch den Ovarien ein Epithel zu, das ich nur bei ersteren finde.

Die Eiersäcke gehen hinten durch die Eileiter in den Uterus itber, von Grenacher und Villot als Uterus, von Vejdovsky als Atrium, von $\mathrm{Cam}$ er a no als Diverticolo cloacale bezeichnet. Gegen die Bezeichnung Uterus dürften keine Bedenken vorliegen, da hier die Befruchtung der Eier vollzogen wird; die die letzteren verkittende Substanz wird auch hier abgesondert, wie ja auch der Sängethier-Uterus die Eihüllen bildet. Nach Camerano soll das Diverticolo cloacale eine Erweiterung des Darms sein, in den die Ausgainge der Geschlechtsorgane eintreten, was sich aber umgekehrt verhält, denn der Darm tritt in das binterste Ende des Uterns.

Die vorderen, symmetrisch getheilten beiden Abtheilungen des Uterus, in welche die Eileiter einmünden, nennt Vejdovsky Hörner des atrium, Camerano rechnet sie nicht zum Diverticolo clvacale, sondern bezeichnet sie als Erweiterungen der Eileiter:

1) I. c. pag. 411 .

2) Zeitschr. für wisscusch. Zoolog. XLVI, 2, Leipzig 1888, pag. 185-216, tab. XVIII. 
Das Receptaculum seminis wurde bereits von v. Siebold ${ }^{1}$ ) und Grenacher richtig erkannt.

Die Cloakenmiindung steht beim Weibchen ebensowenig terminal wie beim Männchen; bei ersterem findet man sie 0,12 mm vom Hinterende entfernt und dahinter zeigt der Körper die Andeutung einer Zweitheilung, wie sie beim Männchen so stark ausgebildet ist.

\section{Anatomie der geschlechtsreifen Thiere.}

Die geschlechtsreifen Exemplare von Gordius tolosanus baben, was bisher übersehen ist, Ocellen. Dicht hinter dem Scheitelpunkt, $0,066 \mathrm{~mm}$ von demselben entfernt, liegen an der Riickenseite, deren Cutis bier noch hyalin, d. h. noch nicht braun gefärbt ist, 2 kleine von schwarzen Pigmentkijgelchen umgebene Linsen in einem Abstand von $0,082 \mathrm{~mm}$ von einander (Fig. 25 a). Die kleinen Pigmentgruppen lassen sich auf Längs- und Querschnitten schon bei schwachen Vergrösserungen erkennen.

Die Entwicklung der Geschlechtsorgane konnte ich an einer Anzahl ganz junger Gordien untersuchen, welche mein Sohn im Juni in einem stagnirenden, fast vertrockneten Waldbache in der Nähe von Northeim fand; es waren 9 Männchen und 2 Weibchen von Gordius tolosanus; die Farbe war hellbraun und das kleinste Männchen hatte nur eine Länge von $68 \mathrm{mn}$, das grösste von $150 \mathrm{~mm}$. Da der Fund während meiner Abwesenheit von Göttingen gemacht wurde, brachte der Finder die Exemplare zu Herrn Dr. Hamann, welcher die Freundlichkeit hatte, nicht nur dieselben za conserviren, sondern auch eine Anzahl ansgezeichneter Serienschnitte anzufertigen, wofür ich an dieser Stelle nochmals meinen verbindlichsten Dank ausspreche.

Die Wandung der beiden an der Rïckenseite der Leibeshöhle symmetrisch neben einander liegenden, die ganze Leibesausdehnung des Männchens der Länge nach durchziehenden Hohlräume der Larve, welche mit einem Epitbel ausgekleidet sind, verwandelt sich in die

Hoden. Das Epithel entspricht weder dem bei den Thieren gewöhnlichen Platten-, Cylinder- oder Flimmerepithel, sondern muss als ein Spindelzellenepithel bezeichnet werden, denn die

1) Archiv für Naturgesch., 1843, pag. 307. 
Ueb. d. Entwicklungsgeschichte u. d. Anatomie v. Gordius tolosanus Duj. 263

Zellen sind spindelförmig, haben einen spindelförmigen Kern und liegen nicht immer mit ihrer Seitenfläche der Wandung an, sondern ragen oft, mit dem einen spitzen Ende in ihr wurzelnd, frei in das Lumeu hinein. Die das Lumen der Hohlräume begrenzende Sulicht des Zellkïrpers wandelt sich in dichtes, gekerntes Bindegewebe um, welches physiologisch dem Hoden entspricht (Fig. 26 a), denn aus ibm sprossen in das Lumen Zellen hinein (Fig. 15), in denen sich Tochterzellen bilden und in letzteren entstehen die Samenkörperchen, welcle bald frei werden und in grossen Massen ansammengeballt in dem Hohlraum nach binten gelangen. Die Epitlelzellen sind die samenbildenden Elemente und an den Stellen dor Wandung, an welchen man die Spermatogenese verfolgen kunn, feblen die ersteren. Dic Spermatogenese vollzieht sich in den jungen, im Wasser lebenden Männchen scheinbar von hinten mach vorn, d. h. man findet bei einem und demselben Männchen im vorderen Drittel des Körpers noch gar keine samenbildenden Zollon, wallrend im mittleren solche in reicher Menge vorbanden sind und im hinteren die Hohlrïume der Hoden bereits von Samenmasse gilnzlich erfullt sind. Die Entwicklung scheint also hinten im Korper zu beginnen und allmäblicb weiter zu schreiten. Die samenbildenden Zellen entwickeln sich aus den Spindelzellen-Epithelien in der Weise, dass aus einer Spindelzelle eine gekernte, gestielte, kugelförmige Zelle wird (Fig. 26c); aus einer solchen entsteht eine grössere, hyaline Zelle mit schwach gefürbtem Kern (d), der dann grösser, stärker gefärbt und granulirt wird (e); in diesen Zellen bilden sich als Mutterzellen Tochterzellen (f), welche in ihrem Innern die Samenkörperchen ausbilden (g).

Die Samenkörperchen sind kurze, dicke Stäbchen mit einer dïnneren und einer dickeren Hälfte; sie sind als Zellen mit excentrischem Kern aufzufassen, und zwar ist der dünnere Theil der chromatische Kern, der dickere der achromatische Zellleib (Fig. 32).

Die Hoden sind bis jetzt noeh von keinem der Forscher, die sich mit Gorlien beschäftigt haben, yesehen worden. Me is sner ${ }^{1}$ ) bezeichnet die Samenmassen als Hoden; Vejdovsky ${ }^{2}$ ) giebt an, es sei ihm nicht gelungen, in den freilebenden Gordien die Hoden in ihrer ursprünglichen Lage und Vertheilung zu entdecken;

1) 1. c. pag. 104.

2) Zur Morphologie der Gordiiden, pag. 417. 
Cameranot) sagt in ähnlicher Weise: "negli esemplari adulti non mi venne fatto di trovar nulla che rivelasse la vera ed intima struttura degli elementi ghiendolare destinati alla produzione degli spermatozoi“" nur Vill $0^{2} \mathrm{t}^{2}$ ) sagt, er habe über den männlichen Geschlechtsapparat in seinen Arbeiten aus den Jahren 1874 und 1881 eine description très exacte et très complète gegeben. In den Nouvelles recherches ans dem Jahre 1881 finden wir (pag. 5) nur die kurze, unichtige Notiz, dass der Same des Männchens vor der Reife entleert wird; in der Monographie des Dragonneaux aus dem Jahre 1874 aber sagt er (pag. 195 und 223) (die die weiblichen Sexualorgane betreffenden Worte sind fortgrelassen): "Les testicules sont deux gros tubes, dans l'intérieur desquels on trouve des spermatozoïdes; ils ne produisent les cellules spermatogènes; ils enveloppent et protègent les éléments essentiels de la reproduction. - Une masse considérable de cellules embryonnaires, autour du tube digestif, se divise en deux parties; l'une centrale, qui se transforme directement en cellules spermatagènes, l'autre périphérique, qui fournit le tissu des testicules". Das verhält sich aber nicht so; die Samenkörperchen entsteben nicht aus Zellen des Zellkörpers; sie bilden sich an der Wand der Hoden, wenn diese bereits Hoblräume sind und keine einzige Zelle des Zellkörpers in ibrem Innern enthalten; sie entstehen aus den wandständigen Epithelzellen. Vill ot hat also die functionirenden Hoden auch nicht gesehen und hält ${ }^{3}$ ) die wahren Hoden für die Hüllen der samenbildenden Zellen.

Aeussere Copulationsorgane, wie Vejdovsky ${ }^{4}$ ) sie beschreibt und abbildet, habe ich bei der von mir untersucbten Art in keinem Falle gefunden; die Bursa balte ich für verbärtete Spermamasse, den Cirrus für ein Kunstproduct.

Die Geschlechtsorgane des erwachsenen weiblichen Thiers bestehen ansser dem bei Beschreibung der Larve erwähnten Uterus sowie dem Receptaculum seminis aus den gleichfalls genannten 5, den Körper der ganzen Lïnge nach durchsetzenden,

1) Ricerche etc., pag. 49.

2) Anatomie des Gordiens, pag. $: 0 \%$.

3) Auatomie des Gordiens, pag. $20 \bar{T}$.

4) Zur Xorphologie etc., tab. XV, Fig, 8 be; tilb. XVI, Fig. 46 el. 
Ueb. d. Entwicklungsgeschichte u. d. Anatomie v. Gordius tolosanus Duj. 265

parallel neben einander verlaufenden Röhren, den beiden Ovarien, den beiden Eiersïcken und dem Ritckencanal.

Die Ovarien sieht man bei jungen Weibchen als prall mit Zellen gefillte Organe (Fig. 27, 28, 29 b); sie beginnen dicht hinter dem Kopfe und werden bald so mächtig, dass sie die Seitenhailften des Körpers derart erfüllen, dass zwischen ihnen nur cin schmaler Raum bleibt, der von den anfangs leeren Eiersilcken, der Leibeshöhle mit dem Darm und dem Bauchnervenstrang eingenommen wird (Fig. 29).

Die Entwicklung und Fortleitung der Eier vollzieht sich in schr merkwilrdiger Weise; die Ovarien füllen sich prall mit gekcrnten Eizellen, welche sich als Tochterzellen in Mutterzellen (Fig. $27 \mathrm{~b}$ ) bilden und sich bald polygonal an einander abplatten; obgleich nun die parallel mit und nach innen von ihnen verlau. fenden Eileiter (Fig. 27, 28, 29 a) selber keine Eizellen produciren, fullen sich diese doch mit der fortschreitenden Entwicklung des Weibchens mit solchen, was in der Weise geschieht, dass an bestimmten Stellen die Scheidewand zwischen beiden Röbren schwindet und die Eizellen aus den Ovarien in die Eileiter hineinwuchern (Fig. 30), wie Vejdorsky ${ }^{1}$ ) diese Communication schon beobachtet hat. In dieser Periode ist ein Ruickencanal noch nicht entwickelt.

Ein ganz anderes Bild gewähren alte Weibchen. Hier beobachtet man, wie die eibildenden Drïsen nur an der Innenwand der Ovarien, da wo Eiersäcke und Leibeshöhle an einander grenzen, ihren Sitz haben (Fig. 31 b), und wie die Eier an der Innenseite der Ovarien entstehen, $u m$ in deren Hohlraum zu fallen, den Vejdovsky mit Leibeshöhle oder Cölom bezeichnet. Bei diesen alten Weibchen sieht man also 6 parallele Hoblränme, in der Banchgegend die Leibeshöhle mit dem Darm, nach dem Schwanzende zu auch das Receptaculum seminis enthaltend (Fig. $31 \mathrm{f}$ ), seitlich die Ovarien (a u. b), zwischen ihnen nach der Rückengegend zu die leeren Eileiter (c) und in der Rückenlinie den von Vejdovsky ${ }^{2}$ ) gefundenen Rückencanal, über dessen Function er unklar ist. Villot ${ }^{3}$ ) nennt ihn richtig einen füften zum Genital-

1) Studien über Gordiiden II, tab. XVIII, Fig. 7, 9, 10.

2) Zur Morphologie etc., pag. 408-409.

3) Anatomie des Gordiens, pag. 201-202. 
apparat gehörigen rudimentären Canal. Bei einem alten Weibchen, dessen Eiablage fast vollendet war, fand ich ibn der ganzen Länge nach mit Eiern gefüllt; bier waren die Lücken in den Wandungeu zwischen Ovarien und Eiersäcken wieder vollständig geschlossen, dieselben besteben also nur zeitweise, und halte ich den Canal fuir einen in diesem Reifestadium in Function tretenden Verbindungsgang zwischen Ovarien und Eiersäcken; am Sehwanzende tritt der Canal von der Riickenlinie etwas nach der Bauchseite zu und wird sehr in die Breite gezogen ${ }^{1}$ ) und die beiden Seitenenden werden in die Hinterenden der Orarien übergehen; der Canal wird nun die Eier nach vorn leiten und sich bier links und rechts in die Eileiter öffnen; welche sie wieder nach binten führen, $\mathrm{um}$ sie in den Uterus zu übertragen. Ich kann bestimmt versichern, bei Thieren, deren Eiablage fast vollendet ist, auf zahlreichen Serienschnitten die Wandung zwischen Ovarien und Eiersäcken stets intact und ohne Oeffnungen gefunden zu haben, wie dasselbe der Fall ist, wenn in ganz jungen Weibchen die Eiersäcke noch leer sind (Fig. 28 u. 29). Wir finden somit vier sebr verschiedene Entwicklungszustände der weiblichen Genitalien, die den Fig. 16, 29, 30 und 31 entsprechen. Begrenzt wird das Ovarium aussen von einer einzelligen Schicht (Fig. 31), einem Rest des Zellkörpers, von Vejdovsky als Epithel bezeichnet.

Die Orarien reicben bis dicht an das Kopfende heran; die Eier entstehen, wie die Samenkörperchen im Hoden, zuerst im hintersten Theile des Ovarium, dann allmählich fortschreitend weiter vorn. Die erste Anlage der Ovarien bestent aus $0,026 \mathrm{~mm}$ grossen, kugelförmigen Zellen, den erwähnten Mutterzellen der Eizellen (Fig. 27).

Verwandt sind die Gordien einerseits mit den Annulaten durch die Segmentirung des Zellkörpers und der Ovarien, durch die Duplicität der männlichen Organe und den an der Bauchseite verlaufenden Nervenstrang, anderefseits aber durch ibre von Camerano ${ }^{2}$ ) beschriebene Embryogenie mit den Nematoden.

1) Vejdorsky. 1. c. tab. XVI, Fig. 64 c.

2) I primi momenti della evoluzione dei Gordii, Torino 1889. 
Ueb. d. Entwicklungsgeschichte u. d. Anatomie v. Gordius tolosanus Duj. 267

\section{Erklärung der Abbildungen auf Tafel XIV-XVI.}

Die Figuren 1-14, 16-24 bezichen sich auf Larven aus Käfern. 15, 25-32 auf im Wasser gefundene Exemplare.

Fig. 1. Scheitelpartie des Kopfondes.

Fig. 2-6 Kopfende, a Kopfganglion und Nervenstrang, b Mundbecher und Oesophagus, Querschnitt.

Fig. 7. Lüngsschnitt durch das Kopfende, a Kopfganglion, a Mundbecher.

Fig. 8. Querschnitt durch eine männliche Larve, a Epidermis, b Cutis oder Derma, c Hypodermis, d Muskulatur, e Nervenstrang, f Darm, g Zellkörper, h Leibeshöhle, i Hohlraum der zukünftigen Hoden.

Fig. 9-14, männliches Schwanzende.

Fig. 10a, Muskulatur.

Fig. 11a, Muskulatur, b getheilter Nervenstrang, c Darm, d Vas efferens.

Fig. 15. Junges, freilebendes Männchen, a samenbildende Hodenzellen.

Fig. 16-20. Hinterleibsende der weiblichen Larve.

Fig. 16. a Hohlraum der zukünftigen Eiersäcke, b Leibeshöhle.

Fig. 17. a Eileiter, Ende der Eiersäcke, b Darm, c Uterus.

Fig. 18. a Darm, b Uterus mit pilzförmigen Wucherungen.

Fig. 19. a Darm, b Uterus mit Netzwerk von einzelligen Drüsen.

Fig. 20. Cloakenmündung.

Fig. 21. a Epidermis, b Cutis oder Derma, c Hypodermis, d Muskeln, e Zellkörper, f Epithel. Längsschnitt.

Fig. 22. Längsschnitt durch den Nervenstrang.

Fig. 23. Längsschnitt durch den Darm.

Fig. 24. Querschnitt durch die Muskulatur, a contractile Substanz, b Kern, c Marksubstanz, d Hypodermis. Hämatoxylinfärbung.

Fig. 25. Querschnitt vom Kopfende dicht hinter dem Scheitel. a Ocellen mit einem Pigmentringe.

Fig. 26. Querschnitt durch die Bauchhälfte des Hodens. a Hodenparenchym, $\mathrm{b}-\mathrm{g}$ die fortschreitende Entwickiung der samenbildenden Zellen. 
268 Dr. v. Linstow: Ueb. d. Entwicklungsg. u. d. A nat. v. Gordius tolosan. Duj.

Fig. 27-29 ganz junges, freilebendes Weibchen. a Eiersäcke, b Ovarien. 27 ganz vorn am Kopfende, 28 vom mittleren, 29 vom hinteren Körperdrittel.

Fig. 30. reifes Weibchen. a Eiersäcke, b Ovarien.

Fig. 31. Weibchen bei fast vollendeter Eiablage. a Hohlraum des Ovarium, b Drüsenzellen des Ovarium, c Fiersack, a Rückenkanal mit Fiern, e Leibeshöhle, f Receptaculum seminis.

Fig. 32. Samenkörperchen. 
5.

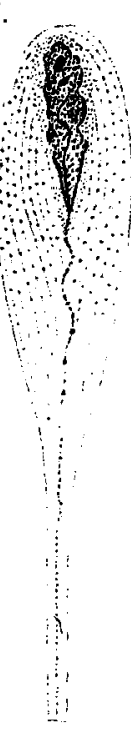

8

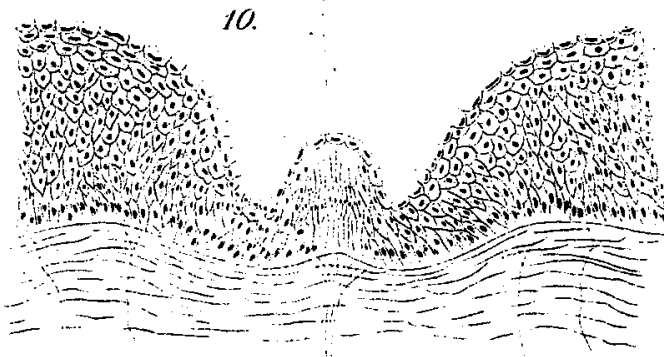

1오.

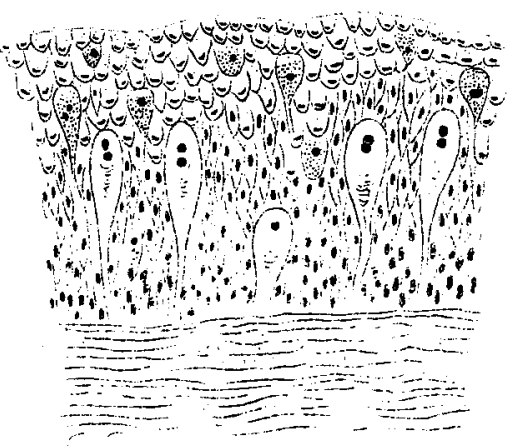

2.

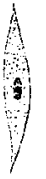

4.

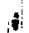

!
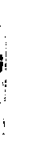

c.
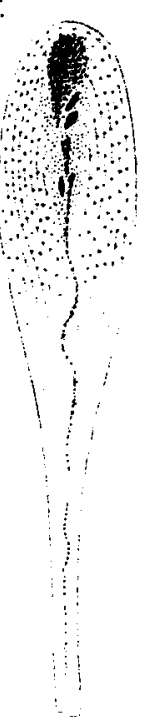




